\title{
BASES MARXISTAS PARA LA EDUCACIÓN AMBIENTAL
}

Guillermo Foladori ${ }^{1}$

\begin{abstract}
Karl Marx has developed a broad theory about the functioning of the capitalist system. He has also given important methodological steps towards analyzing the relationship of the human being with his external environment. This article explains the importance of the concept of universal work developed by Marx. His approach shows the different role that the classes play on the environment and on development.The implications of the evolution of universal work on nature reveal the importance of incorporating the responsibility of social classes to environmental education, not only in terms of the results on contamination and depredation but also to the forces that guide them.
\end{abstract}

Key words: marxism, environmental problems, social metabolism with nature, social classes.

\section{RESUMO}

Karl Marx desenvolveu uma ampla teoria sobre o funcionamento do sistema capitalista. Também deu passos metodológicos importantes para analisar a relação do ser humano com seu ambiente externo. Este artigo explica a importância do conceito de trabalho geral desenvolvido por Marx. Sua abordagem mostra o papel diferente que as classes desempenham na natureza externo e no desenvolvimento. As implicações da evolução do trabalho geral no ambiente externo revelam a importância de incorporar a responsabilidade das classes sociais à educação ambiental, não apenas em termos de resultados de contaminação e depredação, mas também às forças que as orientam.

Palavras chave: Marxismo, questão ambiental, metabolismo com a natureza, classes sociais.

\section{RESUMEN}

Karl Marx ha desarrollado una amplia teoría sobre el funcionamiento del sistema capitalista. También ha dado importantes pasos metodológicos para analizar la relación del ser humano con su ambiente externo. Este artículo explica la importancia del concepto de trabajo general desarrollado por Marx.

\footnotetext{
${ }^{1}$ Universidad Autónoma de Zacatecas (México).
} 
Su enfoque da cuenta del diferente papel que ejercen las clases sobre la naturaleza externa y sobre el desarrollo. Las implicaciones de la evolución del trabajo general sobre el ambiente externo revelan la importancia de incorporar la responsabilidad de las clases sociales a la educación ambiental, no sólo en cuanto a los resultados de contaminación y depredación sino también a las fuerzas que la guían.

Palabras clave: marxismo, cuestión ambiental, metabolismo con la naturaleza, clases sociales.

\section{INTRODUCCIÓN}

Una de las cuestiones más difíciles de entender es la interrelación entre el ser humano y el ambiente externo desde una perspectiva social, más allá de las relaciones técnicas analizadas por las ciencias físico-naturales y biológicas y que explican las causas y consecuencias de la utilizaciones de la tecnología, la producción de químicos, la transformación física del ambiente y el acúmulo de desechos, entre otros efectos perjudiciales. El sentido común, el lenguaje, y la división científica y disciplinar de las ciencias conduce a la distinción entre natural y artificial, creando una dicotomía que las ciencias superan parcialmente al establecer la influencia entre uno y otro polo. Resulta fácil de entender que una persona se contagie debido al contacto con un agente externo a su cuerpo biológico, o que una fuerza natural externa, como un huracán, acabe con un poblado. También resulta fácil de entender que el ser humano afecte el ambiente externo, lo hace con una represa que controla y desvía el curso de un río, y con emisiones de materiales que provocan el calentamiento global. Pero, estos ejemplos son explicados por las ciencias físico-naturales y biológicas. ¿Cuál es el aporte de las ciencias sociales para aquella interrelación entre lo natural y lo artificial? En esto Karl Marx fue mucho más allá de todo lo que han venido planteando las diversas escuelas de economía, sociología, y antropología, por citar las más notorias. Marx mostró un camino muy fructífero para analizar la problemática ambiental, que infelizmente no ha sido continuado salvo excepciones. ${ }^{2}$

\footnotetext{
${ }^{2}$ Existe la creencia equivocada que Marx no se interesó por la cuestión ambiental, o, peor aún, que su discurso era productivista y anti-ambientalista. Para una recuperación meticulosa del pensamiento de Marx respecto de la cuestión ambiental véase J. B. Foster (FOSTER, 2000) y P. Burkett(BURKETT, 1999).
} 
En lo que sigue aunque acotado por el espacio voy a omitir aspectos importantes del análisis de Marx sobre la problemática ambiental que ya fueron ampliamente desarrollados, como la relación con su teoría económica (BURKETT, 1999; BURKETT; FOSTER, 2006), y con el materialismo histórico (BURKETT; FOSTER, 2006; FOSTER, 2000, 2002); me voy a centrar en el aspecto más antropológico del análisis de Marx, poco considerado en los destacados análisis anteriormente mencionados. Este aspecto puede reducirse a las implicaciones ambientales del concepto de trabajo general.

Esta reflexión es importante para la educación y comunicación ambiental debido a que, de otra forma, estas disciplinas terminan repitiendo de forma vulgar consignas mejor desarrolladas por la química, la física y la biología, como que no hay que contaminar, o que hay que producir en forma sustentable. Consignas que son sólo declaraciones de buena voluntad, que dan la impresión que el cambio hacia la sustentabilidad se dificulta por la falta de conocimiento, o el egoísmo individual, que es sólo falta de voluntad. El análisis de Marx, por el contrario, hace énfasis en las relaciones objetivas que emanan del tipo de relaciones sociales de producción y el grado de desarrollo de las fuerzas productivas, y que obligan a un determinado comportamiento con la naturaleza externa de forma necesaria, aunque siempre quede en la decisión colectiva consciente la posibilidad de cambiar el rumbo de las cosas.

\section{EL TRABAJO GENERAL}

El tema que traemos al lector fue planteado por Marx con el concepto de trabajo general. ${ }^{3} \mathrm{El}$ trabajo general es un concepto fuertemente antropológico, que trasciende en buena medida las etapas históricas y los modos de producción, porque corresponde a la esencia de la especificidad humana. El trabajo general es, en breve, el conocimiento. Escribe Marx:

Es trabajo general todo trabajo científico, todo descubrimiento, todo invento. Está condicionado, en parte por la cooperación con seres vivos, y en parte por la utilización de los trabajos de predecesores (MARX, 1978, p. 128).

\footnotetext{
${ }^{3}$ No debe confundirse el trabajo general con el trabajo abstracto, ni con cualquier forma específica de trabajo como el trabajo asalariado, servil, esclavo, etc.
} 
Marx da a entender en la cita anterior que el trabajo general resulta de la cooperación entre los seres vivos y por la acumulación del trabajo histórico. Para entender mejor esta afirmaciónesconveniente algunas aclaraciones.

Primero, el ser humano es resultado de la evolución biológica, y diferencias cuantitativas pueden distinguirlo de sus parientes evolutivos más cercanos, como los simios; pero existe una diferencia cualitativa. Esta diferencia cualitativa consiste en que el ser humano no solamente utiliza útiles e instrumentos para relacionarse con el medio externo, sino que los acumula a través del tiempo. La acumulación de herramientas e instrumentos de generación en generación es lo que permite que sean mejoradas, adaptadas para nuevos usos y socializadas. Muchos animales de diferentes especies utilizan herramientas, pero no las acumulan de generación en generación(FOLADORI; MELAZZI; KILPP, 2016). Esta distinción es clave en el relacionamiento del ser humano con el ambiente externo, ya que el uso de herramientas es el requisito para el metabolismo de la sociedad humana con su ambiente. Marx resume la importancia de los medios de producción para la sociedad humana en la siguiente cita:

\footnotetext{
Ninguna producción es posible sin un instrumento de producción, aunque este instrumento sea sólo la mano. Ninguna es posible sin trabajo pasado, acumulado, aunque este trabajo sea solamente la destreza que el ejercicio repetido ha desarrollado y concentrado en la mano del salvaje(K. Marx, 1972, pp. 7).
}

Este texto, escrito en los Grundrisse(1857-1858) señala el carácter natural de la intermediación entre el ser humano y la naturaleza externa. Además, señala que cada mediación [instrumento] es acumulación de trabajo pasado; trabajo pasado que en otros escritos identifica con el término "trabajo general" o "trabajo universal" -según la traducción.

Segundo, el desarrollo evolutivo de la mano y su íntima relación con el surgimiento del lenguaje han permitido que el Homo sapiens -y tal vez también el $H$. neanderthaly otros- diseñe en la mente lo que va a producir antes de la acción, es decir, tenga una visión de futuro. Muchos animales reaccionan inteligentemente frente a incentivos externos momentáneos, lo cual demuestra una inteligencia práctica, pero para tener una inteligencia teórica es necesario el lenguaje y la visión de futuro. Como escribe Marx, 
AMBIENTE \& EDUCAÇÃO

ISSN- 1413-8638

E-ISSN - 2238-5533

v. 23 , n. 3, p. 159-169, 2018

Una araña ejecuta operaciones que recuerdan las del tejedor, y una abeja avergonzaría, por la construcción de las celdillas de su panal, a más de un maestro albañil. Pero lo que distingue ventajosamente al peor maestro albañil de la mejor abeja es que el primero ha modelado la celdilla en su cabeza antes de construirla en la cera. Al consumarse el proceso de trabajo surge un resultado que antes del comienzo de aquél ya existía en la imaginación del obrero, o sea idealmente(MARX, 1977, p. 216).

Y, también,

Al consumarse el proceso de trabajo surge un resultado que antes del comienzo de aquél ya existía en la imaginación del obrero, o sea idealmente (MARX, 1977, p. 216 T I, Vol 1).

Podemos dividir el proceso de trabajo en dos grandes componentes.Por un lado, el diseño mental; por otro, la actividad. En la realidad ambos se pueden dar simultáneamente e interrelacionados, pero la historia de la humanidad ha ido separando el diseño de la actividad, hasta llegar al capitalismo donde el diseño se separa completamente de la actividad, con la consolidación de la ciencia, de los centros de investigación, y de las universidades que crean conocimiento e inventan productos que luego serán aplicados y producidos por la industria. La más clara evidencia de esta separación entre diseño y producción es la mercantilización del conocimiento. Las diferentes formas de propiedad intelectual permiten que se rente o compre el conocimiento, independientemente de si luego se aplica productivamente 0 se vuelve a vender como patente, por ejemplo.

La pregunta clave que surge de esta evidencia de separación histórica entre diseño mental y actividad práctica es ¿cómo es posible que el conocimiento de la humanidad se acumule de generación en generación; cómo es posible que una generación logre recuperar gran parte del conocimiento pasado, se lo apropie e inclusive lo potencie mejorándolo y adaptándolo a sus necesidades? Esta acumulación histórica y trans-generacional del conocimiento de la humanidad Marx lo llama de trabajo general, porque está en la esencia de la naturaleza humana. Este trabajo general es lo que permite que el ser humano conozca de forma cada vez más profunda las leyes de funcionamiento, leyes físicas, químicas, biológicas, y aplique tal conocimiento en el metabolismo con la naturaleza externa, obteniendo de ella lo necesario para la satisfacción de sus necesidades. Dio Marx respuesta a la pregunta 
anterior al analizar el papel de los medios de producción en el proceso productivo. De entre toda la producción humana, la acumulación y mejora de los medios de producción es el mecanismo más importante para entender el relacionamiento con el ambiente externo,porque son requisito de cualquier proceso productivo. Marx señaló que los medios de producción condensan el conocimiento pasado. Cualquier persona puede utilizar un teléfono inteligente, manejar un auto, o hacer una transferencia bancaria a miles de kilómetros de distancia, sin necesidad de conocer el funcionamiento físico de las ondas electromagnéticas, ni la mecánica elemental de las fuerzas físicas, ni los actuadores incorporados en los sistemas micro-electro-mecánicos. Esto ocurre porque los dispositivos, equipo e instrumentos utilizados han incorporado conocimiento histórico sistematizado y desarrollan actividades automáticamente. Dicho de forma más contundente: la máquina -para ejemplificar cualquier medio de producción-cristaliza, condensa el conocimiento histórico pasado.

Tercero, podemos llamar objetivación del trabajo humano al proceso mediante el cual el conocimiento o trabajo general se deposita en instrumentos, y medios de producción que están por fuera del cuerpo biológico del ser humano, este conocimiento destilado y consolidado en cosas externas se vuelve objetivo, porque está materializado. Una máquina no sólo es un conjunto de piezas, es una articulación de componentes que incorporaron el conocimiento de leyes físicas, químicas y/o biológicas.

Cuarto, existe una enorme diferencia entre el conocimiento dentro de una mente y el conocimiento objetivado en cosas materiales, sean estas libros, computadores, máquinas, o satélites. El conocimiento en la mente no puede ser apropiado más que por su creador y usuario. El conocimiento consolidado en la máquina puede ser apropiado, monopolizado, por personas que no la crearon. Una idea en la mente no puede ser apropiada por otro, una idea escrita en un papel puede ser apropiada por otra persona. Esta objetivación del trabajo humano está en la base del surgimiento y desarrollo de las clases sociales. 
Quinto, la objetivación del conocimiento, desde la más rudimentaria hacha de piedra hasta el más sofisticado microscopio electrónico, es el punto de partida para la existencia de diferencias al interior de la sociedad humana, y para entender la manera como la sociedad humana se relaciona y se distancia en su evolución de la naturaleza externa, pero según la distribución en clases sociales. Como señaló Marx, esa separación entre el ser humano y la naturaleza externa es una separación mediada por los instrumentos.

Esta mediación obliga a un metabolismo muy diferente al resto de los seres vivos; porque en el caso de la sociedad humana el metabolismo depende y se ajusta a la distribución en clases sociales. La apropiación diferencial y clasista de los medios de producción conduce al también desigual metabolismo de las clases con la naturaleza externa. Toda acción sobre la naturaleza externa es, también, una transformación al interior de la propia sociedad humana: "Al operar por medio de ese movimiento sobre la naturaleza exterior a él y transformarla, transforma a la vez su propia naturaleza" (MARX, 1977, p. 215-216 T I, Vol 1).

Por ello lo que las ciencias sociales deben de explicar no es el relacionamiento inmediato "original" y directo de la sociedad humana con la naturaleza externa, sino el proceso mediante el cual se distancia clasistamente. Nuevamente Marx:

Lo que necesita explicación, o es resultado de un proceso histórico, no es la unidad del hombre viviente y actuante, ... con las condiciones inorgánicas, naturales, de su metabolismo con la naturaleza, ... sino la separación entre estas condiciones inorgánicas ... y esta existencia activa, (MARX, 1972, p. 449 T I).

Los cinco aspectos antes desarrollados, esto es: el metabolismo con la naturaleza externa mediado por instrumentos, la separación entre diseño mental y actividad, la objetivación del trabajo humano, la potencial monopolización del conocimiento, y, el metabolismo con la naturaleza externa ajustado a la división en clases sociales, nos permiten ahora abordar el concepto de trabajo general. 


\section{REFLEXIONES SOBRE EL TRABAJO GENERAL PARA LA EDUCACIÓN AMBIENTAL}

En el apartado anterior hemos dado los elementos esenciales para entender la problemática ambiental de forma crítica, tal cual lo hizo Marx. Esos elementos no explican las particularidades de la problemática ambiental en un contexto determinado, ni siquiera bajo relaciones capitalistas (FOLADORI, 2001). Pero, aquellos elementos esenciales sí explican los siguientes aspectos:

- La objetivación del conocimiento en los medios de producción permite su monopolio-aunque no lo haga forzoso-, lo que históricamente ha dado origen a las clases sociales. Todo conocimiento objetivado tiene un origen y un contexto clasista, no neutral, por tanto el accionar de la sociedad humana en relación al ambiente externo debe ser analizado en dicho contexto clasista. No existe un comportamiento ser humano naturaleza, existe un comportamiento de cada clase y sector de clase con la naturaleza externa, que es esencialmente diferente, porque diferente es su lugar en relación con los medios de producción que es la mediación del metabolismo.

- La sociedad humana metaboliza con la naturaleza externa mediante el uso de medios de producción como característica esencial e inevitable de su propia naturaleza, lo cual significa que los responsables de cualquier uso insustentable de los recursos y espacios naturales y/o de la naturaleza transformada son, en primerísima instancia, las clases dueñas de esos medios de producción. Los consumidores, por su parte, son marginales en la responsabilidad sobre la transformación de la naturaleza, y por ende sobre la problemática ambiental; y la educación ambiental debiera transmitir esta importante diferencia objetiva de responsabilidad social.

- El conocimiento depende crecientemente de medios objetivados. No existe hoy en día ciencia que pueda desarrollarse sin laboratorios, equipos sofisticados, computadoras, softwares y demás. Como este instrumental también es potencialmente monopolizable, y lo está en realidad, la clase capitalista no solamente es responsable principal por el uso de los medios de producción sino también por el uso y destino de la ciencia, incluida la 
ISSN- 1413-8638

E-ISSN - 2238-5533

v. 23 , n. 3, p. 159-169, 2018

ciencia orientada a mitigar y reencauzar los problemas ambientales. Como escribió Marx,

El desarrollo del medio de trabajo como maquinaria no es fortuito para el capital, sino que es la metamorfosis histórica del medio de trabajo legado por la tradición, transformado en adecuado para el capital. La acumulación del saber y de la destreza, de las fuerzas productivas generales del cerebro social, es absorbida así con respecto al trabajo, y por el capital y se presenta por ende como propiedad del capital, y más precisamente del capital fixe, en la medida en que éste ingresa como verdadero medio de producción al proceso productivo..." (MARX, 1972, p. 220 Tomo II).

Como resalta Marx en la cita, el capital se apropia de la ciencia, es decir de la quintaesencia del trabajo general, por tanto de la acumulación histórica de saber que ella contiene y, necesariamente, del devenir de dicha ciencia, con lo cual también de la orientación de la educación, que es el paso previo al desarrollo científico. Una educación ambiental crítica debe tomar distancia de las explicaciones técnicas para resaltar las explicaciones sociales.

\section{CONCLUSIÓN}

En los párrafos anteriores se ha llamado la atención del lector sobre un concepto del instrumental teórico de Marx poco divulgado, y menos utilizado para entender aspectos puntuales como el de la problemática ambiental. Este concepto es el de trabajo general. El trabajo general es el conocimiento de la humanidad acumulado a través de los siglos.

Debido a la especificidad del ser humano como productor de instrumentos, el relacionamiento con el medio ambiente externo es crecientemente mediado por equipo, maquinaria, infraestructura, y dispositivos de diferente tipo según la actividad. Todo esto es conocimiento que ha sido destilado de las leyes físico químicas y biológicas y cristalizado en medios objetivos, externos al ser humano. Son estos medios de producción el mecanismo esencial que tiene el ser humano para transformar y adaptar a la naturaleza para satisfacer las necesidades sociales. El metabolismo del ser humano con la naturaleza externa depende del tipo, amplitud y ritmo con que se utilizan los medios de producción. 
Como estos medios de producción pueden y son monopolizados, en nuestra sociedad por la clase capitalista, resulta que es ésta la responsable en primerísima instancia de los problemas ambientales.

Es común que la educación ambiental exprese las preocupaciones más aparentes sobre aspectos como la contaminación o la depredación del medio ambiente; pero, con el instrumental teórico que ha legado Marx es posible ir aún más lejos, identificando las causas y responsables últimos de la crisis ambiental contemporánea.

\section{REFERENCIAS}

BURKETT, P. Marx and Nature.A Red and Green Perspective. New York: St. Martin's Press, 1999.

BURKETT, P.; FOSTER, J. B. Metabolism, energy, and entropy in Marx's critique of political economy: Beyond the Podolinsky myth. Theory and Society, v. 35, n. 1, p. 109-156, 1 fev. 2006.

FOLADORI, G. Limites do desenvolvimento sustentável. Campinas, SP: Editora da Unicamp, 2001.

FOLADORI, G.; MELAZZI, G.; KILPP, R. A economia da sociedade capitalista e suas crises recorrentes. Sao Paulo: Outras Expressões, 2016.

FOSTER, J. B. Marx's Ecology. Materialism and Nature. New York: Monthly Review Press, 2000.

FOSTER, J. B. Ecology Against Capitalism. [s.I.]Monthly Review Press, 2002. MARX, K. Elementos fundamentales para la crítica de la economía política: Grundrisse. 1857-1858. 3 Tomos. Traducao José Aricó; Miguel Murmis; Pedro Scaron. México: Siglo XXI, 1972.

MARX, K. El Capital. Tomo I, Vol. 1. El proceso de producción del capital. Traducao Pedro Scarón. México: Siglo XXI, 1977. 
AMBIENTE \& EDUCAÇ̃̃o

ISSN- 1413-8638

E-ISSN - 2238-5533

v. 23 , n. 3 , p. $159-169,2018$

MARX, K. El capital. Tomo II, Vol. 5. Traducao Pedro Scarón. México D.F.:

Siglo XXI, 1978. 